\title{
Multi-fractal analysis and lacunarity spectrum of the dark matter haloes in the SDSS-DR7
}

\author{
C. A. Chacón-Cardona ${ }^{a, b, *}$, R. A. Casas-Miranda ${ }^{a}$,J.C. Muñoz-Cuartas ${ }^{c}$ \\ ${ }^{a}$ Grupo de Astrofísica, Facultad de Ciencias, Universidad Nacional de Colombia, Ciudad \\ Universitaria, Carrera 30 No. 45-03, Bogotá D.C., Colombia. \\ ${ }^{b}$ Grupo AstroUD Sabio Caldas, Facultad Tecnológica, Universidad Distrital Francisco \\ José de Caldas, Transversal 70B No. 73a-35 sur, Bogotá, D.C., Colombia \\ ${ }^{c}$ FACom-Física-FCEN, Universidad de Antioquia, Calle 70 No. 52-21, Medellín, \\ Colombia
}

\begin{abstract}
The dark matter halo distribution of the nearby universe is used to study the fractal behaviour in the proximate universe. The data, which is based on four volume-limited galaxy samples was obtained by Muñoz-Cuartas and Mueller (2012) from the Seventh Data Release of the Sloan Digital Sky Survey (SDSS-DR7). In order to know the fractal behaviour of the observed universe, from the initial sample which contains 412468 galaxies and 339505 dark matter haloes were used as input for the fractal calculations. Using this data we use the sliding-window technique for the dark matter distribution and compute the multi-fractal dimension and the lacunarity spectrum and use it to study its dependence on radial distance in every sample.

The transition to homogeneity is not observed in the dark matter halo distribution obtained from the SDSS-DR7 volume-limited galaxy samples; in its place the dark matter halo distribution exhibits a persistent multi-fractal behaviour where the measured dimension does not arrive at the value of the physical dimension of the space, for all structure parameter values of the analysed set, at least up to radial distances of the ordered from $165 \mathrm{Mpc} / \mathrm{h}$ from the available centres of each sample. Our results and their implications are discussed in the context of the formation of large-scale structures in the
\end{abstract}

\footnotetext{
${ }^{*}$ Corresponding author

Email addresses: cachaconc@unal.edu.co (C. A. Chacón-Cardona ), racasasm@unal .edu.co (R. A. Casas-Miranda), jcmunoz@fisica.udea.edu.co (J.C. Muñoz-Cuartas)
} 
universe.

Keywords: Fractals, Cosmology, Statistical physics PACS: 05.45.Df, 98.80.-k, See 05

\section{Introduction}

The current cosmological view asserts that the universe is homogeneous and isotropic (Peacock, 1999; Martínez and Saar, 2002; Longair, 2008; Wald, 2010); the observed heterogeneities are local in nature and should vanish at sufficiently large scales(Grujić, 2003; Gabrielli et al., 2005; Komatsu et al., 2009). This principle is based firstly on philosophical considerations: the observed universe must be statistically equal to any observer regardless of the point and direction of observation; and secondly, on cosmological observations: mainly isotropy measurements of the cosmic microwave background (Caruso and Oguri, 2009; Kobayashi et al., 2011), clustering analysis of galaxies and observations of Type Ia supernovae (Riess et al., 1998; Perlmutter et al., 1999; Hamuy et al., 2000; Hamuy, 2013). This standard cosmological principle constitutes the basis of cosmology as a branch of physics. From the structure of space-time of the universe, tolarge-scale structure formation, this principle is present in the conceptual foundations, in the statistical information processes and the interpretation of the results.

However, this approach excludes possible solutions that do not assume the homogeneity hypothesis, such as models based on the Lemaitre-TolmanBondi metric (Bondi, 1947; Célérier, 2000; Enqvist, 2008) or fractal relativity models based in the conditional cosmological principle proposed by Benoit Mandelbrot, in his words:

The distribution of galaxies is the same, as seen of any point that belongs for this distribution.

(Mandelbrot, 1983, 1997) where the universe appears to be statistically the same from every galaxy-like occupied point of the fractal set (Not in void regions), and in every direction. Some models that have in account this conditional principle are refereed in Ribeiro (1992); Humphreys et al. (1998); Mittal and Lohiya (2003); Uchaikin (2004) .

Despite the success of the physical models constructed from this cosmological principle, there are still fundamental questions without answer in the context of the structure formation in the universe. Some researchers 
now are debating heavily whether the observations from more recent galaxy catalogs corroborate the assumption of homogeneity and isotropy at scales greater than $60 \mathrm{Mpc} / \mathrm{h}$ (Hogg et al., 2005; Sarkar et al., 2009; Capozziello and Funkhouser, 2009; Bagla et al., 2008; Scrimgeour et al., 2012) or if instead the galaxies are grouped into highly structured hierarchical patterns, with properties of fractality (Coleman and Pietronero, 1992; Borgani, 1993; Durrer and Sylos Labini, 1998; Gabrielli et al., 2005; Gaite, 2005, 2007; Sylos Labini et al., 2009; Verevkin et al., 2011).

Hogg et al. (2005) used 55000 Red Luminous Galaxies from the first data release of the SDSS to study the homogeneity of this sample at redshift $z \approx 3$, in this work they found, by correlation analysis, the homogeneity transition around of $70 \mathrm{Mpc} / \mathrm{h}$. Sarkar et al. (2009) corroborated these results in their multi-fractal analysis of SDSS DR6, where they found a transition to homogeneity in the same spatial region. Yadav et al. (2010) established theoretically the upper limit of the homogeneity transition around the 260 $\mathrm{Mpc} / \mathrm{h}$ for a $\Lambda \mathrm{CDM}$ numerical model. Similarly, Scrimgeour et al. (2012) used the fractal correlation dimension to analyse data of galaxy catalogues in the southern sky. From this, they conclude that the transition to homogeneity takes place on the spatial scale around $80 \mathrm{Mpc} / \mathrm{h}$ using in their analysis the fractal correlation dimension.

In this work, we aim to study the multi-fractal dimension and the corresponding lacunarity spectrum based on the generalised correlation integral for a dark matter haloes distribution found on four volume-limited samples of galaxies in the Seventh Sloan Digital Sky Server Data Release (SDSS DR7) (Abazajian et al., 2009; Muñoz-Cuartas and Mueller, 2012), using depurated information which was provided by the New York University Value-Added Galaxy Catalog (NYU-VAGC) (Blanton et al., 2005). One of the novelties of this work rest on the use of dark matter halos identified from a galaxy redshift survey, instead of the galaxies by itself. We argue that using our halo catalogs instead of galaxies leads to a better estimate of the fractal dimension of the observable Universe since neither galaxy bias nor strong redshift space distortions have to be considered in the analysis.

The multi-fractal dimension spectra, a powerful statistical tool to provide a characterisation of highly irregular distributions of objects was successfully applied by the authors to determine the homogeneity transition, the radial dependence of the fractal dimension and lacunarity spectrum in the Millennium Simulation dark matter haloes (Springel et al., 2005; Chacón-Cardona and Casas-Miranda, 2012). Now, we must see if the real Universe behaves 
as simulations do, the same methodology is applied on the best galaxy data available and is presented in order to evaluate its behaviour from the multifractal point of view the observed Universe.

This paper is organised as follows. In section 2, the mathematical foundations involved in the determination of multi-fractality and the lacunarity spectrum of dark matter clustering is reviewed. Section 3 contains a summary of the characteristics of DR7, the volume-limited samples of galaxies and the corresponding dark matter distributions that we use. In section 4, the calculations of multi-fractality and lacunarity are discussed for the case of dark matter clustering up to a redshift of 0.2 . In section 5 , the results of multi-fractality and lacunarity are discussed, and the conclusions are presented in section 6 .

\section{Theoretical Formalism}

Fractal analysis of the galaxy distribution is based on the idea of generalising the dimension of a metric space. This concept, developed by Hausdorff (1918), allowed Benoit Mandelbrot to define a fractal as a set with Hausdorff dimension strictly exceeding its topological dimension (Mandelbrot, 1983), where the topological dimension is the number of independent directions that can be taken around an element belonging to a given set. It is possible to determine the Hausdorff dimension for finite sets using computational algorithms, such as the minimum spanning tree (MST). However, in the case of the galaxy distribution, the correlation dimension $D_{2}$, is a very useful tool for measuring the fractality in the galaxy clustering on large scales (Martínez and Saar, 2002).

\subsection{Correlation Dimension and Lacunarity}

The large scale matter distribution can be characterised with a fractal spectrum defined from a generalised correlation. First, it is necessary to introduce the correlation integral $C_{2}$, a function capable of measuring the number of neighbours that on average a chosen centre has within a distance $r$. In agreement with Bagla et al. (2008), we define the correlation integral $C_{2}$ as:

$$
C_{2}(r)=\frac{1}{N M} \sum_{i=1}^{M} n_{i}(r)
$$


where $N$ is the total number of particles inside the distribution, $M$ is the number of particles used as centres and the summation is performed over that set $M$ of chosen centres. $n_{i}(r)$ is the number of particles within a radial distance $r$ from the $i$ th-particle, defined as:

$$
n_{i}(r)=\sum_{j=1}^{N} \Theta\left(r-\left|\mathbf{x}_{\mathbf{i}}-\mathbf{x}_{\mathbf{j}}\right|\right),
$$

where the summation is performed over all the particles in the sample. The coordinates of each particle in our space of three dimensions are denoted by $\mathbf{x}_{\mathbf{j}}$, and $\Theta$ is the Heaviside function, defined such that $\Theta(x)=0$ for $x<0$ and $\Theta(x)=1$ for $x \geqslant 0$. The number of particles around each centre $n_{i}(r)$ is determined by counting the number of particles that lie inside a comoving sphere of radius $r$ from the centre.

From Equation (1) the correlation dimension is defined similarly to the definition of mass-radius dimension (Blumenfeld and Mandelbrot, 1997):

$$
C_{2}(r) \approx r^{D_{2}}
$$

So that the correlation dimension is calculated as a derivative:

$$
D_{2}=\frac{d \log C_{2}(r)}{d \log r}
$$

By expressing the correlation integral as a power law, in addition to the multi-fractal dimension, the set is fully characterised by the proportionality constant $F$ that accompanies the function. The pre-factor $F$ which is related to the average distance between the nearest neighbours, permits defining the lacunarity, which is a property that determines how the fractal set fills the space. Defined by Blumenfeld and Mandelbrot (1997) via the second-order variability factor for the pre-factor $F$, the lacunarity is expressed as:

$$
\Phi=\frac{\left\langle(F-\langle F\rangle)^{2}\right\rangle}{\langle F\rangle^{2}}=\frac{\left\langle F^{2}\right\rangle}{\langle F\rangle^{2}}-1 .
$$

Whereas lacunarity was defined as a complementary concept to the fractal mass-radius dimension, this concept can be applied to other definitions of fractal dimension, as shown in (Martínez and Saar, 2002); The correlation dimension and its corresponding lacunarity are sufficient to characterise sets for which these quantities do not depend on the scale, as discussed in the 
majority of studies that demonstrate fractal behaviour, at least on small scales. Therefore, in the case of scale dependence of fractal quantities we must extend the statistical analysis by using the formalism of the multifractal dimension (Nakamichi and Morikawa, 2004; Gabrielli et al., 2005).

\subsection{Generalised Fractal Dimension}

From the correlation integral $C_{2}(r)$, Equation (1), the generalised correlation integral can be defined as:

$$
C_{q}(r)=\frac{1}{N M} \sum_{i=1}^{M}\left[n_{i}(r)\right]^{q-1}
$$

where $M$ is the number of centres, $N$ is the total number of particles included in the sample, $n_{i}(r)$ is the same expression defined in the Equation (2) and $q$ is called the structure parameter, which corresponds to an arbitrary real number. From this generalised correlation it is possible to do an expansion in powers of $\log (r)$ as described by Provenzale et al. (1997) and then calculate directly the multi-fractal dimension and the lacunarity spectrum:

$$
\log \left[C_{q}(r)^{1 /(q-1)}\right]=D_{q} \log (r)+\log \left(F_{q}\right)+O\left(\frac{1}{\log (r)}\right) .
$$

Keeping only the first two terms on the right side, we have the relation between the generalised correlation integral and the generalised fractal dimension:

$$
C_{q}(r)^{1 /(q-1)}=F_{q} r^{D_{q}}
$$

In this manner the generalised dimension and the generalised lacunarity can be defined, the generalised dimension $D_{q}$ :

$$
D_{q}=\frac{1}{(q-1)} \frac{d \log C_{q}(r)}{d \log r},
$$

and the corresponding generalised lacunarity from the pre-factor $F_{q}$ for every structure parameter $q$ :

$$
\Phi_{q}=\frac{\left\langle\left(F_{q}-\left\langle F_{q}\right\rangle\right)^{2}\right\rangle}{\left\langle F_{q}\right\rangle^{2}}=\frac{\left\langle F_{q}^{2}\right\rangle}{\left\langle F_{q}\right\rangle^{2}}-1 .
$$


If for any $q_{1} \neq q_{2}, D_{q_{1}}=D_{q_{2}}$ is verified then it is said that the distribution is a homogeneous fractal (mono-fractal). For $q \geqslant 1, D_{q}$ explores the scaling behaviour in high density environments (clusters and superclusters) and for $q<1$ values, $D_{q}$ explores the scaling behaviour in low-density environments, i.e., voids (Sarkar et al., 2009). In the event that the distribution of dark matter haloes undergo the transition to homogeneity, all values of the fractal dimension must close to the physical dimension of the space $\left(D_{q} \rightarrow 3\right)$ and the spectrum of lacunarity must tend to zero $\left(\Phi_{q} \rightarrow 0\right)$, at the same radial distance $r$.

Next, we apply the concepts of generalised fractal dimension and lacunarity to the dark matter haloes distribution obtained from the Sloan Digital Sky Server data, in its seventh release of the galaxies information.

\section{Dark Matter Haloes from the seventh data release of SDSS in the NYU-VAGC catalog}

Computing the fractal analysis of the mass distribution in the large scales from the distribution of galaxies may have different inconveniences. First of all, galaxies are a biased tracer of the mass density field. In this sense, care must be taken to account for the bias of the galaxy population used to trace the mass distribution. Usually this bias is an unknown function that depends in a complex way on structure and galaxy formation process and then has to be modelled, something that in principle would make our fractal estimates dependent on the bias model in use. Secondly, galaxy redshift surveys like SDSS do not provide information to get the real-space coordinates of galaxies. Since distance to galaxies in redshift surveys is estimated through redshift, the distribution of galaxies in such a data set may be strongly affected by peculiar motions, enforcing an analysis in redshift space. This redshift-space distortions affects the geometrical distribution of galaxies in small and intermediate scales, and for the estimation of fractal dimension this may introduce a significant bias. In order to avoid the problems induced by the use of galaxies, we decided to use instead the information associated to the dark matter halos hosting these galaxies. Using the information of haloes may lead to avoid the necessity of modelling galaxy bias, since all selection effects may be included already in the halo identification process, and secondly and more important, for the effects of the spatial distribution, working with halos would lead us to avoid strong redshift space distortions, 
which would lead to a better and real estimation of the fractal dimension of the mass distribution in the large scales.

In order to avoid the aforementioned inconveniences that appear when working with galaxy data, we will use dark matter haloes drawn from the galaxy data in the SDSS-DR7. The methods used for the halo identification allow us to ignore galaxy bias and strong redshift space distortions. It is out of the scope of this work to go in to the details of the construction of the dark matter halo catalogs used in this work. The reader may be interested in to look for all the details in Muñoz-Cuartas and Mueller (2012). However, for the seek of completeness we present a brief summary of the main features of the method.

The dark matter halo distribution used for our fractal calculations was estimated by Muñoz-Cuartas and Mueller (2012) from galaxy data of the SDSS-DR7 (Abazajian et al., 2009) using the value added products from the catalog NYU-VAGC (Blanton et al., 2005). The NYU-VAGC catalog consists of 7966 square degrees of spectroscopic coverage with an exhaustive quality revision in photometric calibrations including the best redshift for each object, corrections for fiber collisions with the object distances corrected for peculiar velocities. This catalog offers the best information to carry out large scale structure studies. The galaxy data used as input for the construction of the dark matter halo catalogs is made up of four limitedvolume samples in the redshift range from $\mathrm{z}=0.002$ to $\mathrm{z}=0.2$ and limits in absolute magnitude the first one $M_{V L}^{1}=-23.5$ for all volume limited samples and the second one $M_{V L}^{2}=-18.0,-19.0,-20.0,-21.0$ respectively. Table 1 summarises the properties of each galaxy sample and halo catalogs. The selected objects in NYU-VAGC catalog belongs to the main galaxy sample, with a well resolved spectral target. For each galaxy in the data set we compute the absolute magnitude as:

$$
M_{x}-5 \log h=m_{x}-5 \log \left(D_{L}\right)-25-K(z)-A_{x}\left(z-z_{n}\right),
$$

where $h=H_{0} /\left(100 \mathrm{kms}^{-1} M p c^{-1}\right)=0.72, m_{x}$ corresponds to apparent magnitude limit in every band, $D_{L}$ is the luminosity distance, $K(z)$ is the $\mathrm{k}$ correction estimated as in Blanton and Roweis (2007) and $A_{x}$ determines the correction for evolution proposed by Blanton et al. (2003). The values of the parameters used in the cosmological distances calculations were $\Omega_{m}=0.258$, $\Omega_{\Lambda}=0.721, \Omega_{R}=8.493 e-5$, and $\omega_{0}=-1$.

The dark matter halos used to make the multi-fractal analysis were iden- 
tified in the four volume limited samples in accordance with the identification method proposed by Muñoz-Cuartas and Mueller (2012). Differently from other methods, there is no assumption of any ad-hoc parameter, nor linking length or density threshold. The algorithm starts with the association of one dark matter halo for every galaxy within the sample and with its mass calculated from the galaxy luminosity or stellar mass. With this initial mass the properties of dark matter halo were calculated (mass and radius); In the next step the set of nearest galaxies in an ellipsoidal region was searched which axes are calculated by the virial radius and its maximum circular velocity. Groups of galaxies that were intercepted by the ellipsoidal region of given halo were joined into the group in a similar manner to the Friends-of-Friends method with the difference in that linking length is of local character and only depends of the halo properties where the search is made. To the reader interested in the calculations details we recommend the article (Muñoz-Cuartas and Mueller, 2012).

In order to make a fractal analysis of the dark matter haloes identified in the volume limited samples, we should determine the radius of the largest sphere in which fractality calculations are performed without introducing assumptions about the shape of the clustering to be analysed. The effective depth for every sample according to their limits in right ascension and declination is calculated by the equation (Gabrielli et al., 2005):

$$
R_{s}=\frac{R_{d} \sin (\delta \theta / 2)}{1+\sin (\delta \theta / 2)},
$$

where $R_{d}$ corresponds to the maximum radial distance of the sample and $\delta \theta=\min \left(\alpha_{2}-\alpha_{1}, \delta_{2}-\delta_{1}\right)$ are the limits in right ascension of the sample, and $\delta_{1}$ and $\delta_{2}$ are the limits in declination. The algorithm used to calculate the correlation integral selects the centres in accordance with the effective depth over every sample such a way the centres near of borders are not taken in account. The summary of the data samples used in our fractal calculations is presented in Table 1

Figure 1 shows the comoving $(x ; y)$ projection of the different samples. As it can be seen in the Figure 1, most of the redshift space distortions have been strongly reduced due to the use of haloes instead of galaxies in the volume of the survey (Muñoz-Cuartas and Mueller, 2012). Using just the positions of the galaxies in the parent catalog would have lead to the introduction of geometric defects associated with redshift space distortions that would bias our estimates of fractal dimension and lacunarity. 


\begin{tabular}{cccccccc}
\hline \hline Sample & $z_{\max }$ & $M_{V L}^{2}$ & $N_{\text {gals }}$ & $N_{h}$ & $R_{s}$ & $N_{c}$ & $\bar{n}$ \\
\hline$M r-18$ & 0.047 & -18.0 & 50986 & 38268 & 52.4 & 7178 & $1.74 \times 10^{-2}$ \\
\hline$M r-19$ & 0.074 & -19.0 & 108546 & 85222 & 81.9 & 13341 & $1.07 \times 10^{-2}$ \\
\hline$M r-20$ & 0.115 & -20.0 & 155890 & 128975 & 126.6 & 20378 & $4.33 \times 10^{-3}$ \\
\hline$M r-21$ & 0.175 & -21.0 & 97064 & 87040 & 190.2 & 12651 & $8.66 \times 10^{-4}$ \\
\hline \hline
\end{tabular}

Table 1: Volume limited Samples and Dark Matter Haloes found in SDSS data release 7. The redshift higher limit is $z=0.2$ and $M_{V L}^{1}=-23.5$ is the maximum absolute magnitude for all the samples, $R_{s}$ is the effective depth in the subsample, $N_{c}$ is the number of the available centres to make fractal calculations into the every sample, $\bar{n}=N / V$ is the density number of dark matter haloes where $V$ is the equivalent cubic comoving box volume of every sample.

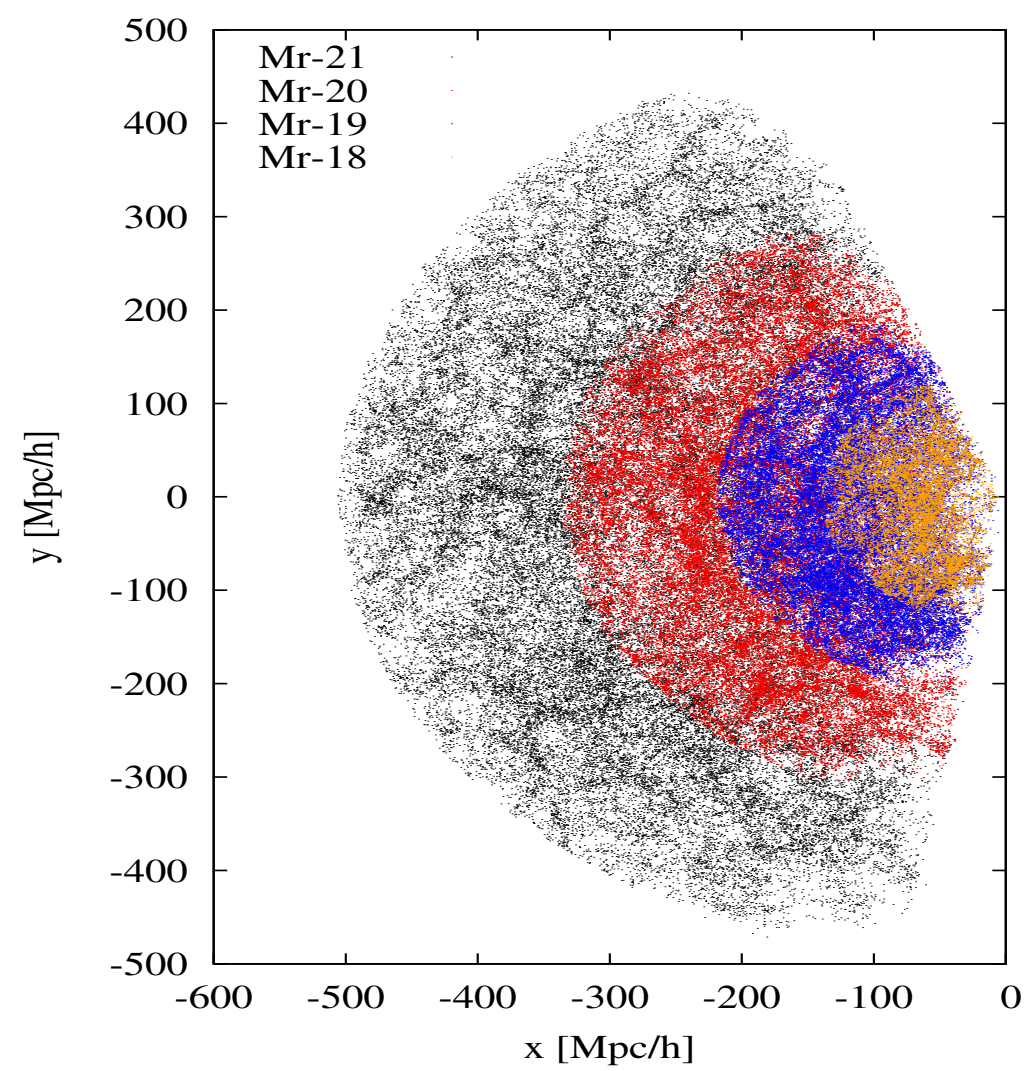

Figure 1: Dark Matter Haloes of the Volume limited samples in SDSS-DR7, the positions of every halo in comoving cartesian coordinates. 
The next step involves the determination of the multi-fractal dimension set and lacunarity spectrum for the subsamples, expecting to answer the question: Is there the homogeneity transition in the dark matter halo distribution as is found in the Millennium simulation by Chacón-Cardona and Casas-Miranda (2012)?

\section{Multi-fractality and lacunarity spectrum for dark matter clus- tering in SDSS-DR7}

In the previous section, we present volume limited samples of galaxies with redshifts in the range of $0.002<z<0.2$. The complete set is composed of 412468 galaxies, with 339505 dark matter haloes identified in the complete sample of galaxies. We calculated the generalised correlation integral, the fractal dimension and the corresponding lacunarity in the dark matter distribution. The uncertainty of $C_{q}$ is determined by the use of error propagation in accordance with the expression:

$$
\Delta C_{q}(r)=\frac{1}{N M} \sum_{i=1}^{M}(q-1)\left[n_{i}(r)\right]^{q-2} \Delta n_{i}(r),
$$

where $n_{i}(r)$ is the number of particles within a radial distance $r$ with respect to a particle $i$ chosen as the centre ( Equation 2 ), $\Delta n_{i}(r)$ is numerically calculated counting the number of dark matter haloes that every radial interval of each centre could be included into the count of $n_{i}(r)$ but they were not included by the uncertainty in their position, i.e., haloes near the borders of the spherical shells used in the count of $n_{i}(r)$.

In order to make the calculations of the correlation integral $C_{q}$ a bin size of $1.0 \mathrm{Mpc} / \mathrm{h}$ was used. The generalised correlation integral was determined for 13 values of the structure parameter in the range $-6 \leqslant q \leqslant 6$, for each dark matter distribution in the corresponding galaxy sample (see Figure 2). These calculations include high-density regions, i.e., $(q \geqslant 1)$, and low-density regions $(q<1)$. For $q=1$, the numerical limit is used.

The fractal dimension spectrum is determined from the generalised correlation integral using the sliding-window technique (Martínez and Saar, 2002). This procedure is applied to the logarithm of the correlation integral as a function of the logarithm of the radial distance. Dividing the curve into segments and making a weighted linear least-squares fit through each set of successive points in a log-log plot we calculate the slope, the intercept and 

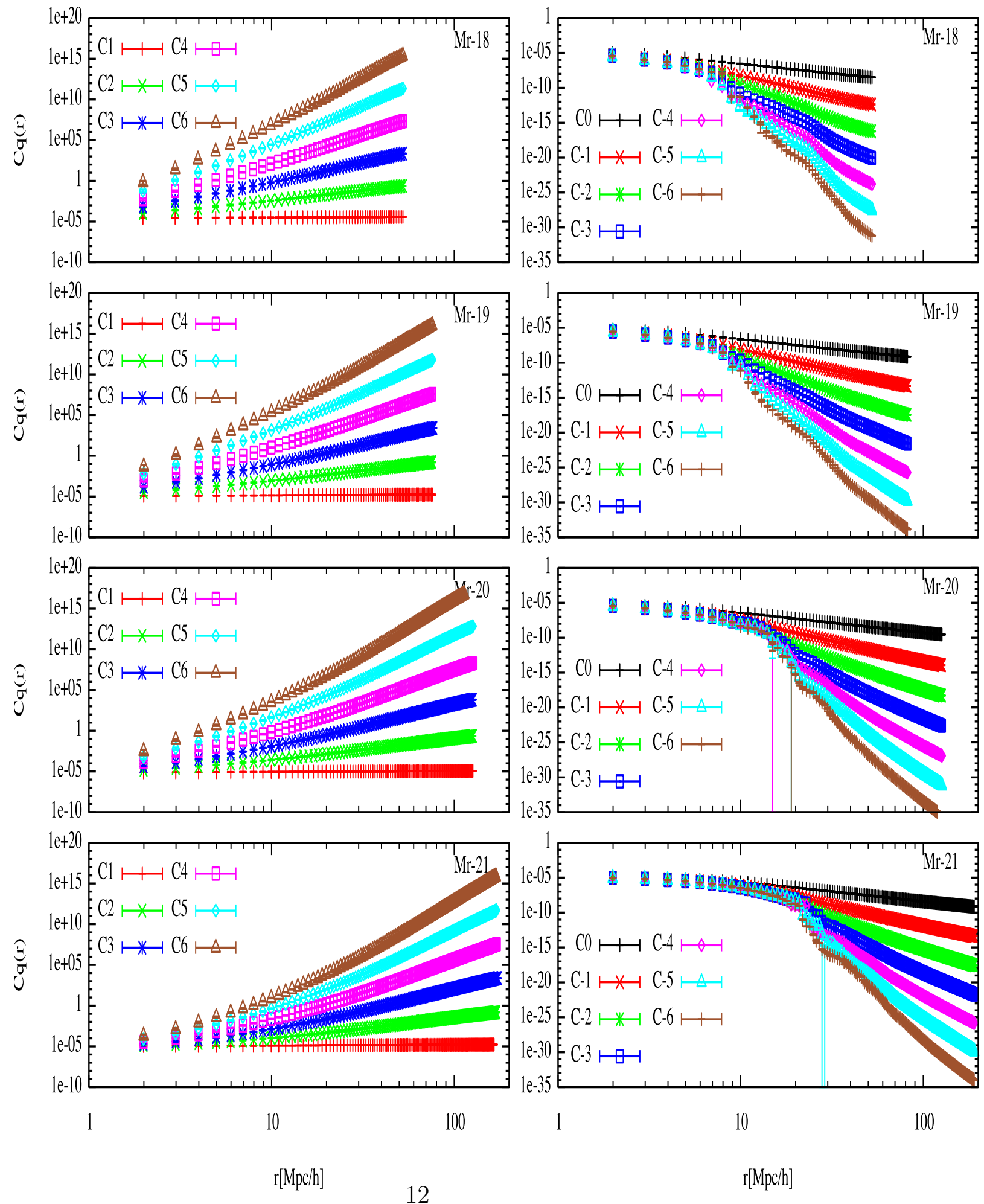

Figure 2: Generalised correlation integral vs. Radial Distance for all values of $q$ studied in this article calculated from the dark matter haloes distribution. Over-densities with $q \geqslant 1$ left, low-densities, with $q<1$, right. The uncertainty $\Delta C_{q}$ in the samples Mr-20 and Mr-21 in two radial intervals are the same magnitude order of the $C_{q}$ and corresponds to the large error bars in log-log scale. 
their associated uncertainties from the covariance matrix in every section of the curve.

Below, Figure 3 shows the results of the fractal dimension spectrum for low-density regions with structure parameter values $q<1$. For high-density regions, namely the correlation integral for structure parameter values in the range $q \geqslant 1$, and the dependence of the spectrum of multi-fractal dimension on radial distance is shown in Figure 5.

These results can be combined to determine the multi-fractal dimension spectrum as a function of the structure parameter $q$ for different radial distance scales for every sample, as shown in Figure 6

Given that the fractal dimension does not specify how galaxies fill space, our analysis is completed by the calculation of the lacunarity spectrum for the same values of the structure parameter. The Figures 8 and 9 show the corresponding results.

\section{Discussion}

In (Chacón-Cardona and Casas-Miranda, 2012) the authors performed a multi-fractal analysis on the clustering of dark matter haloes from the Millennium Simulation (Springel et al., 2005) where they successfully found the homogeneity transition in accordance with standard cosmological principle at depths between $100 \mathrm{Mpc} / \mathrm{h}$ and $120 \mathrm{Mpc} / \mathrm{h}$. We also successfully applied the same methodology to the clustering of dark matter haloes in the local universe using as a basis the dark matter halo distribution obtained by Muñoz-Cuartas and Mueller (2012) from the galaxy distribution in four volume limited samples from SDSS-DR7 (Abazajian et al., 2009), available in the NYU-VAGC catalog (Blanton et al., 2005). We used the comoving distance from FLRW metric in order to locate the dark matter distribution in the scenario of the standard cosmology. The analysed samples were obtained from the best galaxy catalog to carry out statistical studies for large-scale structures, the NYU-VAGC, which presents the most dependable information in completeness and corrections such us fibre collisions; besides, the use of dark matter halo distributions allowed us to compare our results with those obtained in the fractal analysis of the Millennium dark matter simulation, with the advantage that the majority of the redshift space distortions have been strongly reduced in order to avoid bias in our multi-fractal calculations.

From a set of 333505 dark matter haloes identified in four volume limited samples of galaxies with redshifts in the range $2 \times 10^{-3}<z<0.2$, we found 

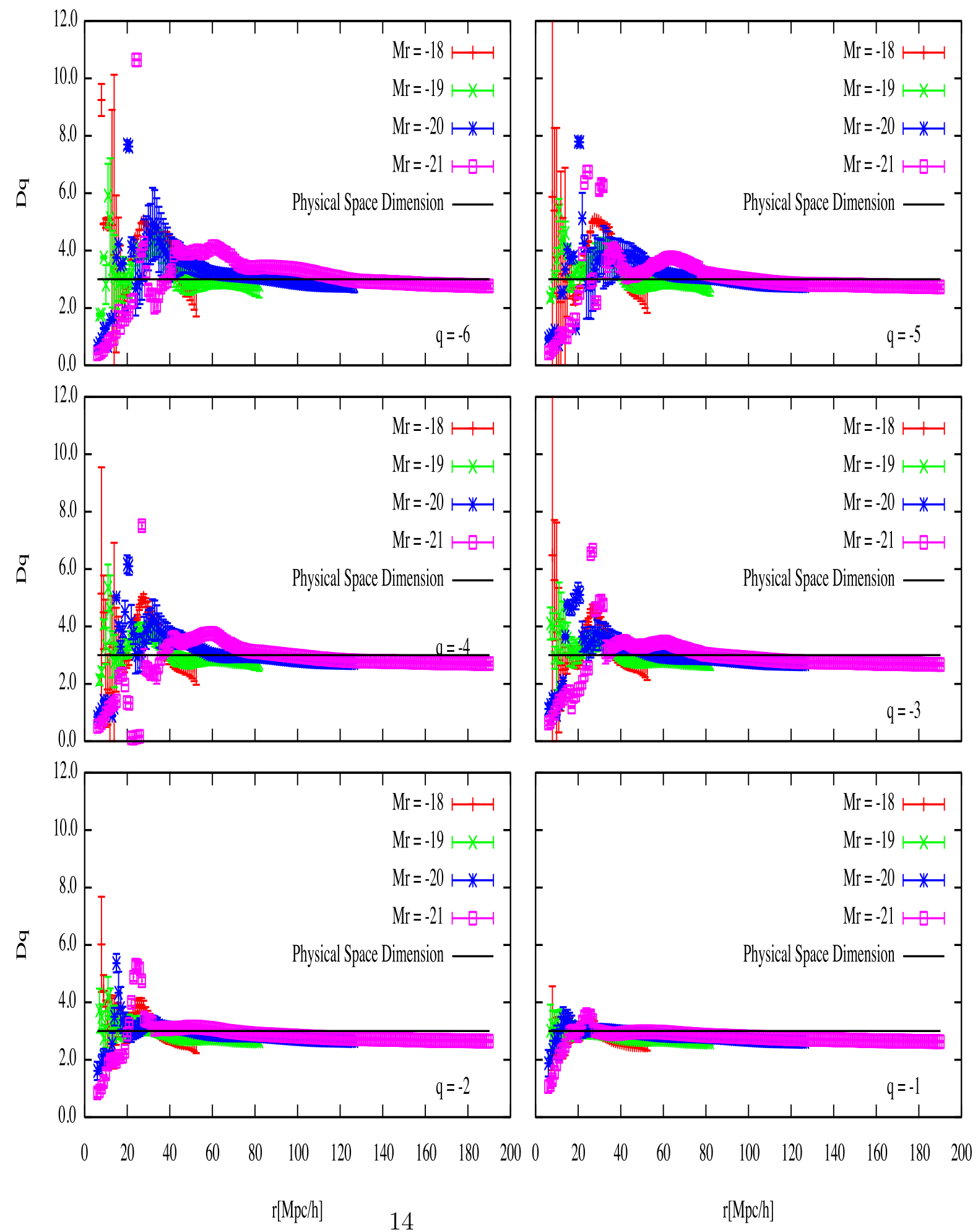

Figure 3: Multi-fractal spectrum $D_{q}(r)$ as a function of the radial distance $r$ for lowdensity environments, with $-6 \leqslant q \leqslant 1$, for dark matter distribution in the four volume limited samples, from SDSS DR7. $\pm 1 \sigma$ error bars in the graphics, with 0.99 confidence level in t-student test. 


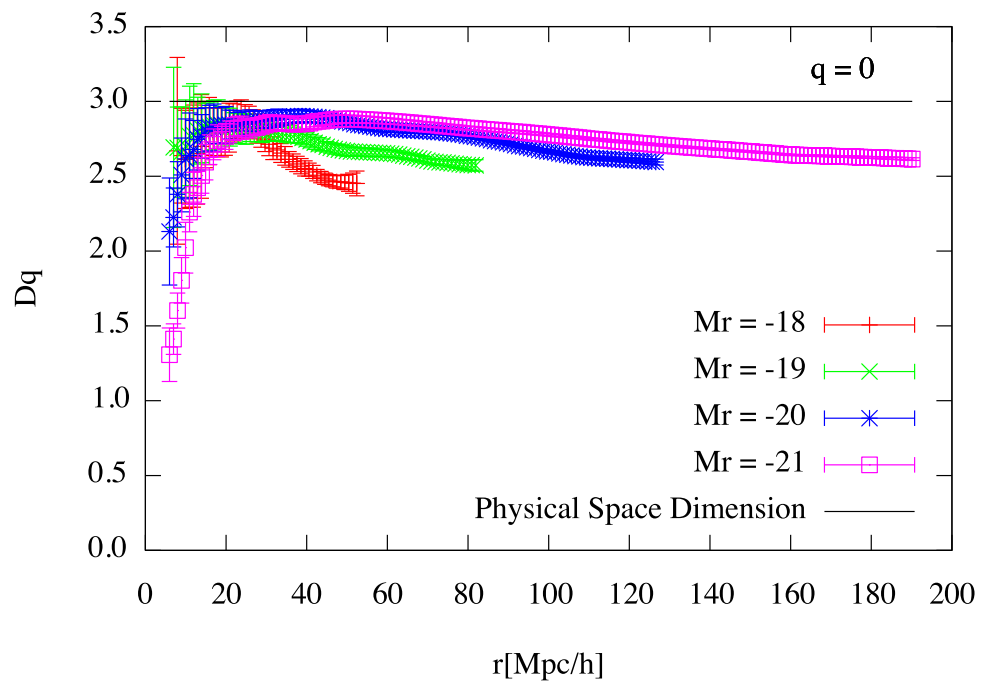

Figure 4: Multi-fractal spectrum $D_{q}(r)$ as a function of the radial distance $r$ for lowdensity environments, with $q=0$, for dark matter distribution in the four volume limited samples, from SDSS DR7. $\pm 1 \sigma$ error bars in the graphics, with 0.99 confidence level in t-student test.

the fractal behaviour of the dark matter clustering. In the calculations that we performed, we took into account the the maximum radius of the sphere that we can inscribe within the limits of our sample using the comoving distance, for which the fractal behaviour could be analysed in every chosen subsample, (Gabrielli et al., 2005) and using only as the centres of the dark matter haloes whose distance to the edges of each sample were greater than or equal to the effective depth in order to avoid edge effects (The same technique was used in the calculations for the Millennium simulation).

We calculated directly the multi-fractal dimension using the generalised correlation integral in the dark matter samples with radial depths reaching even to $190 \mathrm{Mpc} / \mathrm{h}$, beyond the region where we expected to find a clear homogeneity transition. In general terms, the set of integrals found for dark matter clustering behaves as we expected in consistency with the results of (Chacón-Cardona and Casas-Miranda, 2012) and (Sarkar et al., 2009). The resulting functions indicate an increase in the average number of neighbours that are located around the centres in high-density regions, for which $q \geqslant 1$, and a decrease in the number of neighbours for low-density regions $(q<1)$.

Using the sliding-window technique, the fractal dimension for low-density 

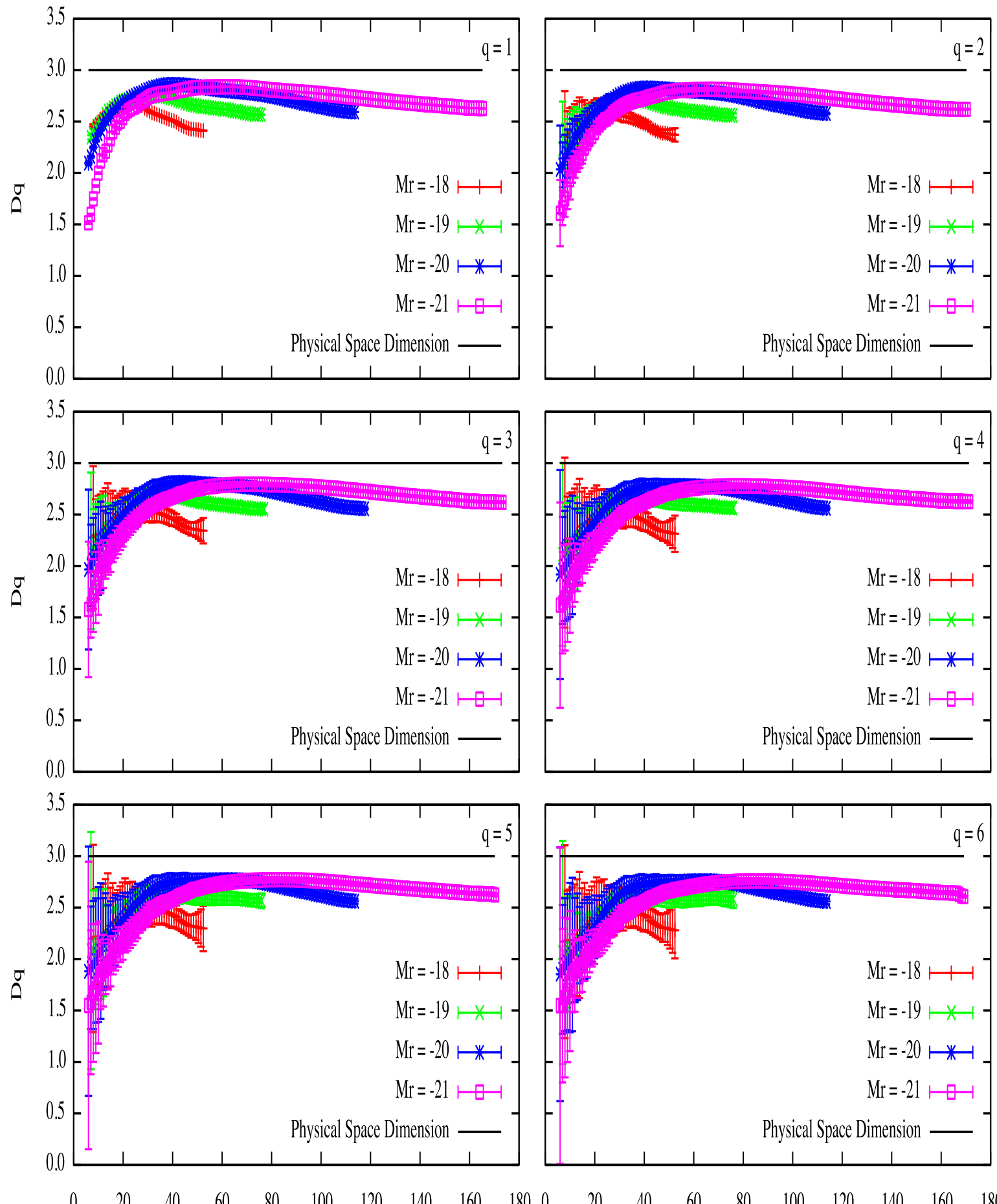

$\mathrm{r}[\mathrm{Mpc} / \mathrm{h}]$

$\mathrm{r}[\mathrm{Mpch}]$

Figure 5: Multifractal spectrum $D_{q}(r)$ as a function of the radial distance $r$ for highdensity environments, with $1<q \leqslant 6$, for every dark matter distribution in the four volume limited samples, from SDSS DR7. $\pm 1 \sigma$ error bars in the graphics, with 0.99 confidence level in t-student test. 

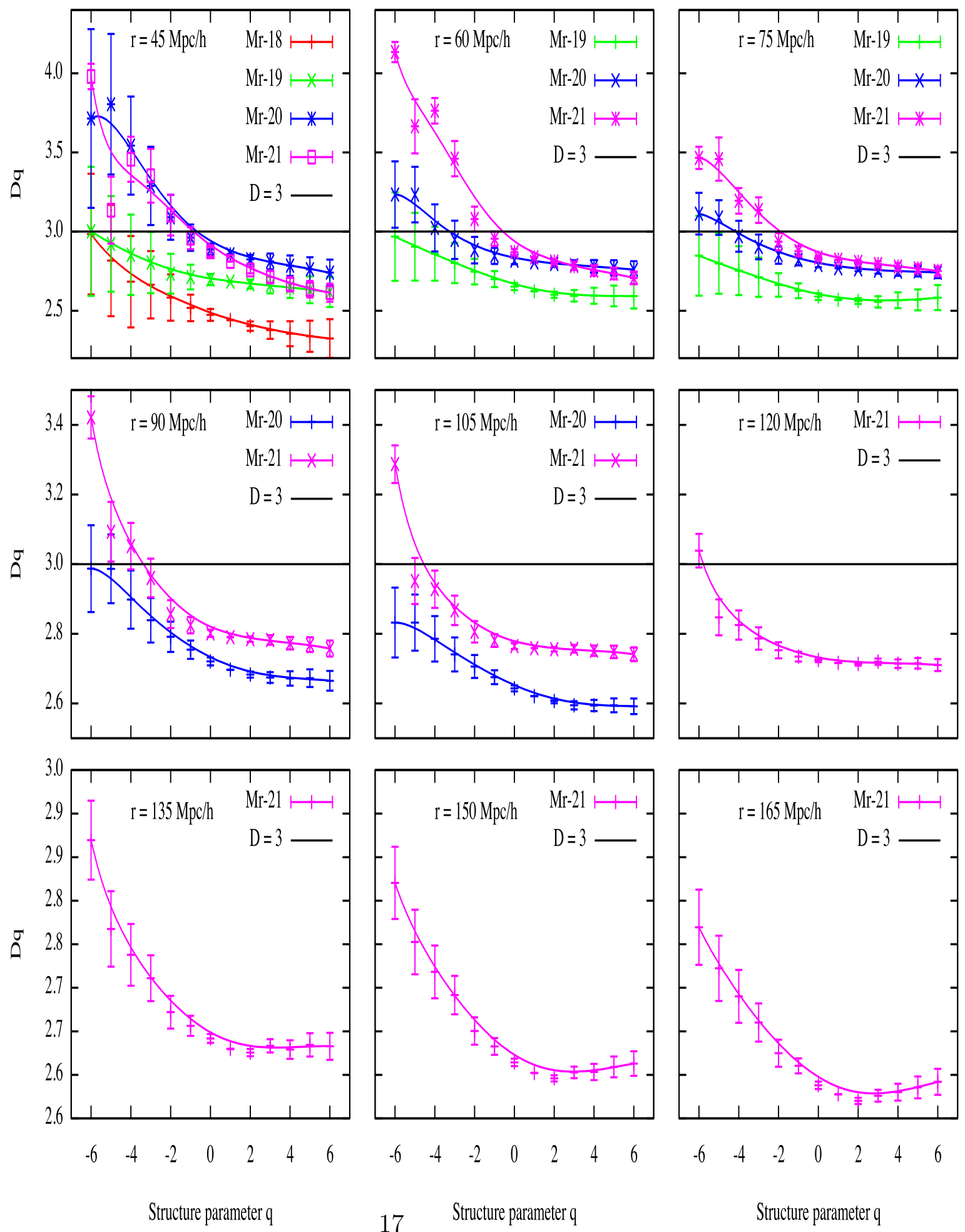

Figure 6: Multi-fractal dimension spectrum $D_{q}(r)$ of the dark matter haloes distribution as a function of the structure parameter $q$ in the range $[-6,6]$. The multi-fractal behaviour is observed even at depths of the order of $165 \mathrm{Mpc} / \mathrm{h}$ around the centres. The solid line indicates the Bézier interpolation curve. 

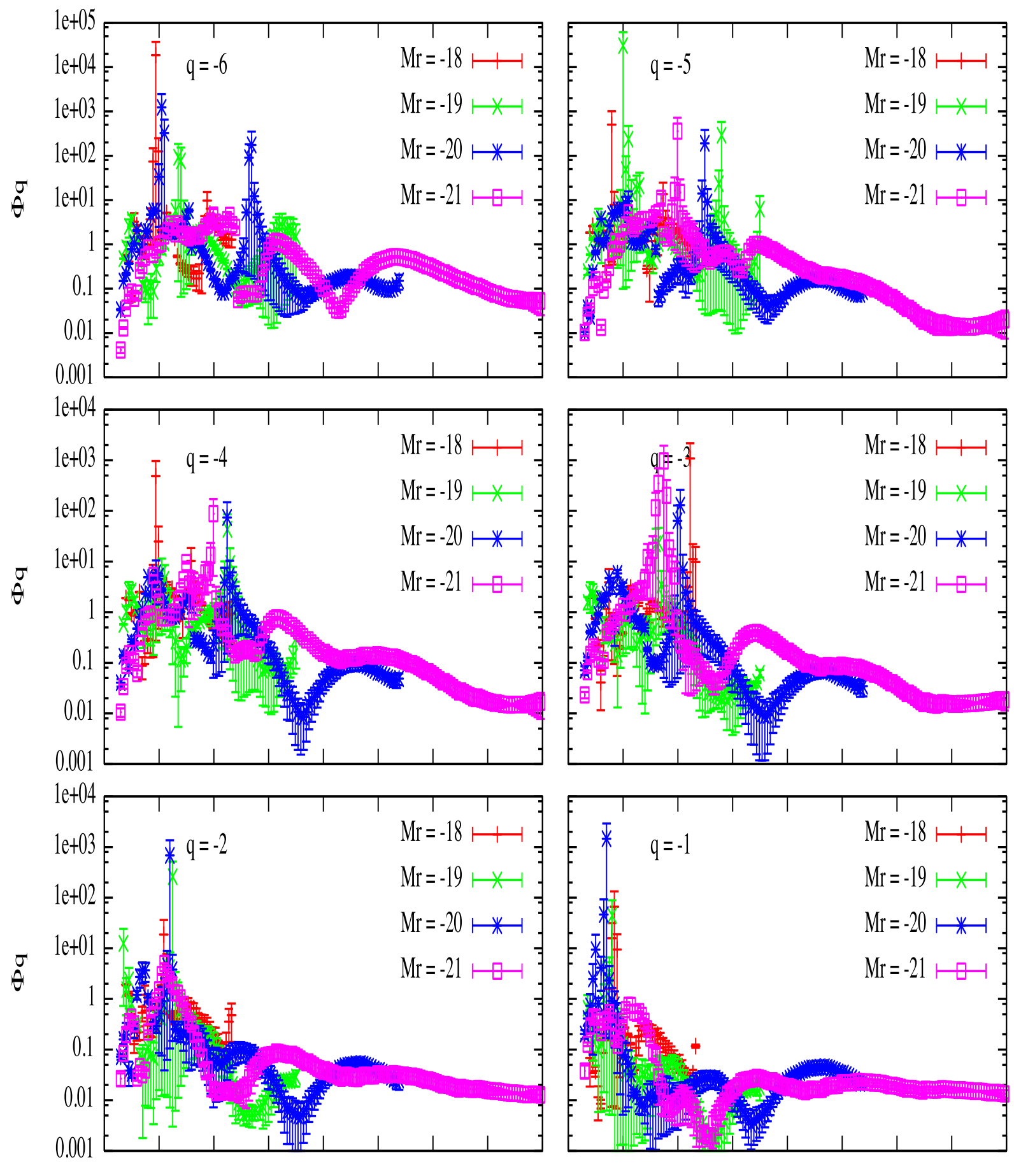

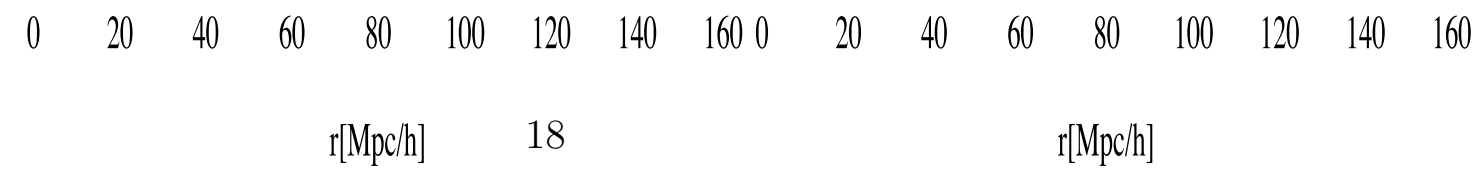

Figure 7: Lacunarity spectrum $\Phi_{q}(r)$ in logarithmic scale as a function of the radial distance $r$ for low-density environments, with $-6 \leqslant q \leqslant 1$, for every dark matter distribution from the four volume limited samples, in SDSS DR7. Error bars of $\pm 1 \sigma$ are indicated in the graphs. 


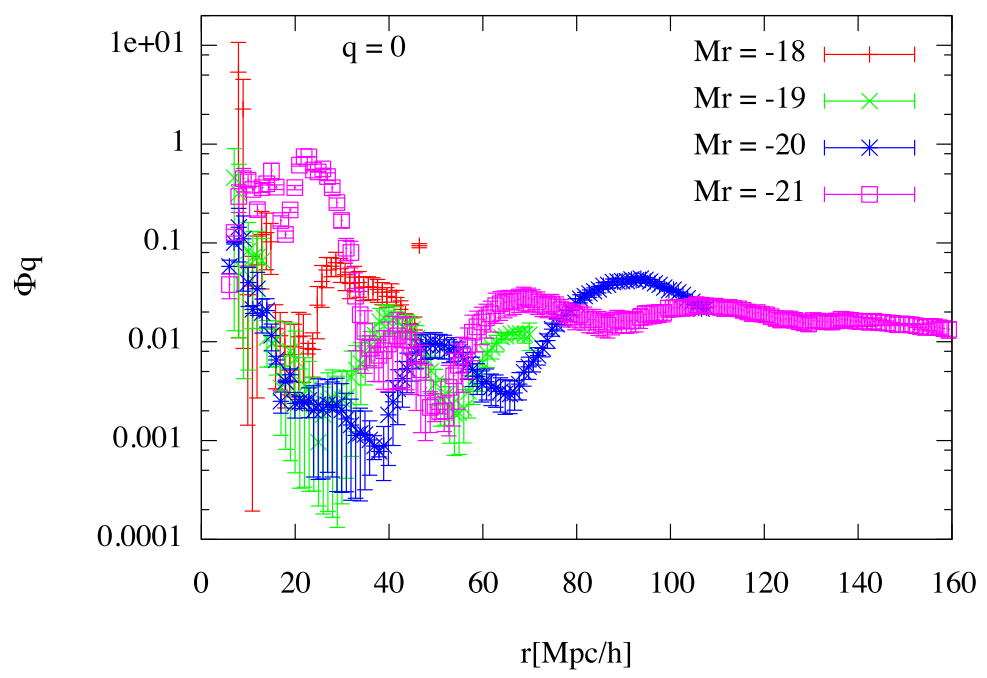

Figure 8: Lacunarity spectrum $\Phi_{q}(r)$ in logarithmic scale as a function of the radial distance $r$ for low-density environments, with $q=0$, for every dark matter distribution from the four volume limited samples, in SDSS DR7. Error bars of $\pm 1 \sigma$ are indicated in the graphs.

regions with structure parameter values $q<1$ were calculated. In accordance with the findings for the Millennium Simulation (Chacón-Cardona and CasasMiranda, 2012), we found a growth that surpassed the physical dimension of space in regions near the centres, but in contrast, after that region we did not find a clear trend towards homogeneity with fractal dimension values that deviate from the physical space dimension.

For high-density regions with structure parameter values $q \geqslant 1$, the behaviour also differs from that which we found previously in the Millennium simulation (Chacón-Cardona and Casas-Miranda, 2012) and the results of (Hogg et al., 2005), (Sarkar et al., 2009), (Scrimgeour et al., 2012). Although there is a region of rapid dimensional growth, asymptotic behaviour towards the physical dimension of space is not observed in all the multi-fractal dimension spectrum.

The dimension functions observed for the $D_{q}$ spectrum with $q<1$ values shows us that the space is almost completely filled (on average) by lowdensity regions (for depths larger that the homogeneity scale), while the persistent fractal behaviour of $D_{q}$ spectrum for $q \geqslant 1$ values indicates that the high-density regions are grouped in small space regions with auto-similarity 

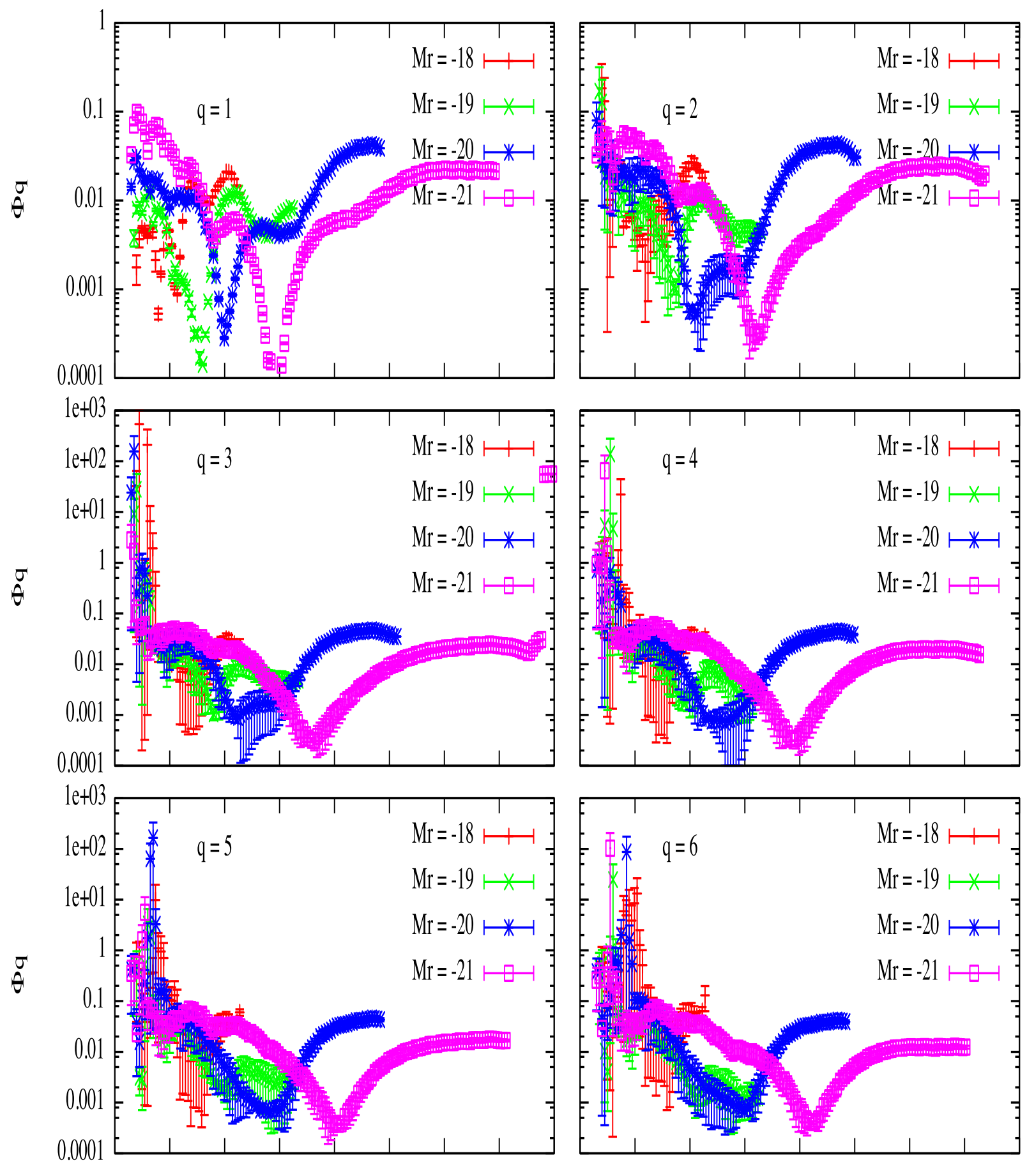

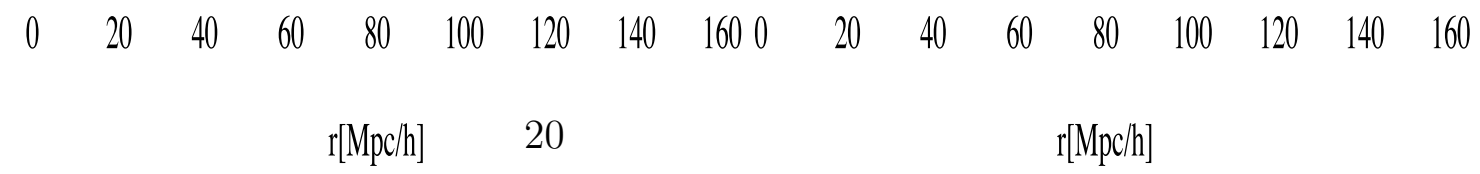

Figure 9: Lacunarity spectrum $\Phi_{q}(r)$ in logarithmic scale as function of the radial distance $r$ for high-density environments, with $q>1$, for dark matter distribution from the four volume limited samples, in SDSS DR7. Error bars of $\pm 1 \sigma$ are indicated in the graphs. 
properties corresponding to galaxy clusters. In this way the universe would present two different behaviours, one for low-density regions and another for regions occupied by galaxies, which would favour the conditional cosmological principle.

In our analysis, the multi-fractal dimension reaches a high value in regions around the centres, followed by a second region where the dimension of the galaxy clustering tends to values that differ from the physical dimension of space; in the region near the centres we observe a great variability, represented by high error values, which grows as the structure parameter does, followed by a tendency to a constant value both in dimension like in its uncertainty with a tendency to reach a constant value between 2.5 and 3 without reaching 3 . The fractal dimension value reported in the previous works is near to 2 , a dimension value less that our dimension measures for $q \geqslant 1$. In accordance with Ribeiro (2005) this behaviour is present in the radial calculations because of the use of comoving distance from FRW metric by the effect of the standard cosmological principle.

This behaviour shows a persistent multi-fractal distribution at larger scales. The multi-fractality is observed most clearly in the sigmoid curves in Figure 6 where it is evident a higher dimensionality (closest to the physical dimension of the space) in voids for all the radial distances. In contrast with the behaviour of dark matter clustering, the fractal dimension values move away from the physical dimension of the space in all cases. Thus, the dark matter distribution does not reach homogeneity, since all dimensions of the multi-fractal spectrum have to accomplish the condition of homogeneity. This finding is consistent with the dark matter clustering behaviour on large scales, as published by Joyce et al. (2005) and Verevkin et al. (2011), and also it is consistent with studies of the anisotropy of the cosmic background radiation realised by Kobayashi et al. (2011).

The determination of the lacunarity spectrum is spoiled by the propagation of errors, inherent in its calculation based on the pre-factor $F$. Despite this difficulty, we analysed first the spectrum of lacunarity of low-density regions $(q<1)$, observing an oscillatory behaviour from initial low values (high homogeneity near the centres) with increasing values (inhomogeneity tendency) until it reached the average maximum value. The lacunarity of high-density environments $(q \geqslant 1)$ also shows an oscillatory behaviour starting with high values at small radial distance (high inhomogeneity near the centres) and then a decreasing at distances a homogeneity tendency in the regions between $60 \mathrm{Mpc} / \mathrm{h}$ and $90 \mathrm{Mpc} / \mathrm{h}$ returning to a final increase at the 
radial limit of every subsample. These results are consistent with deviations from the transition to homogeneity detected by the multi-fractal dimension spectrum.

In order to confirm that the behaviour found in the previous analysis were not the result of difficulties in calculating fractality, two tests were performed. In the first test, the minimum mass scale used to define the population of dark matter haloes of the Millennium simulation was changed with basis on the minimum values of mass of each of the samples analysed from SDSS DR7. It was found that the definition of the population of haloes not affect the results obtained previously by Chacón-Cardona and Casas-Miranda (2012). In the second test, the fractal analysis was performed in a square box of $300 \mathrm{Mpc} / \mathrm{h}$ side, which was extracted from dark matter haloes on Mr-21 sample (SDSS DR7 data). In this case it was verified that the condition of homogeneity is not reached despite having changed the geometry of the sample.

\section{Conclusions}

In the case of dark matter haloes found in the SDSS seventh release, no transition to homogeneity was observed in the dark matter clustering, at least for scale of distance around $165 \mathrm{Mpc} / \mathrm{h}$; instead, there is a clear persistence of multi-fractal behaviour. For values of the structure parameter corresponding to low densities $(q<1)$, the calculated dimensions exhibited a tendency to fill space close to the homogeneity (dimension values very close to the physical dimension of the space) at scales larger than $60 \mathrm{Mpc} / \mathrm{h}$.

For structure parameter values $q \geqslant 1$, i.e. high density environments, the fractal-dimension spectrum showed two clearly defined regions: a first region of high-dimensional growth on scales less than $45 \mathrm{Mpc} / \mathrm{h}$ and a second region of smooth decay to a constant fractal dimension below the physical dimension of the space. Therefore, the use of multi-fractal analysis is most suitable for the characterisation of dark matter clustering because a monofractal dimension analysis could show a homogeneity transition tendency, but this really has not been happened due to the multi-fractal behaviour being dependent on the scale and the structure parameter $q$. Thus, the multi-fractal dimension spectrum offers us a wide vision of dark matter clustering, where the homogeneity transition is only reached if the complete set of multi-fractal dimension reaches the physical dimension of the space.

The lacunarity spectrum confirmed the persistence of the fractal behaviour of the set, showing us the two different ways in which the voids 
and the clusters of galaxies fill the space respectively. The low density regions tend to fill the space almost homogeneously from small scales while the galaxy set shows a decreasing behaviour with alternation between high and low lacunarity values, i.e., the presence of voids followed by regions of high galaxy clustering. We did not observe an absolute lacunarity decrease that would otherwise allow detecting homogeneity. On the contrary we confirmed the departure from homogeneity within the analysed volume limited samples, based on the growth of the lacunarity function.

The dark matter distribution is eminently multi-fractal. A tendency towards mono-fractality (i.e., only one fractal-dimension value independent of the structure parameter) was not observed in the volume limited samples for the available depths used in the analysis. This result implies that further detailed research is needed to continue investigating the standard cosmological principle and its consistency with the observations. Although we cannot conclude that a transition to homogeneity is not found at greater depths, the problem of introducing homogenisation due to cosmological assumptions should be analysed, with the goal of approaching this problem without introducing biases contained in the standard cosmological principle.

\section{Acknowledgements}

C. A. Chacón-Cardona thanks the Universidad Distrital Francisco José de Caldas for their financial support to perform doctoral studies. He also acknowledge the financial support from the Universidad Nacional de Colombia under the "Convocatoria del programa nacional de proyectos para el fortalecimiento de la investigación, la creación y la innovación en posgrados de la Universidad Nacional de Colombia 2013-2015". J.C. Muñoz-Cuartas was suported by "Estrategia de Sostenibilidad 2014-2015 de la Universidad de Antioquia". Funding for the Sloan Digital Sky Survey (SDSS) has been provided by the Alfred P. Sloan Foundation, the Participating Institutions, the National Aeronautics and Space Administration, the National Science Foundation, the U.S. Department of Energy,the Japanese Monbukagakusho, and the Max Planck Society.The SDSS Web site is http://www.sdss.org/. The SDSS is managed by the Astrophysical Research Consortium (ARC) for the Participating Institutions. The Participating Institutions are The University of Chicago, Fermilab, the Institute for Advanced Study, the Japan Participation Group, The Johns Hopkins University, Los Alamos National Laboratory, the Max-Planck-Institute for Astronomy (MPIA), the Max-Planck-Institute 
for Astrophysics (MPA), New Mexico State University, University of Pittsburgh, Princeton University, the United States Naval Observatory, and the University of Washington.

\section{References}

Abazajian, K. N., Adelman-McCarthy, J. K., Agüeros, M. A., Allam, S. S., Allende Prieto, C., An, D., Anderson, K. S. J., Anderson, S. F., Annis, J., Bahcall, N. A., et al., Jun. 2009. The Seventh Data Release of the Sloan Digital Sky Survey. ApJS182, 543-558.

Bagla, J. S., Yadav, J., Seshadri, T. R., Oct. 2008. Fractal dimensions of a weakly clustered distribution and the scale of homogeneity. MNRAS390, 829-838.

Blanton, M. R., Hogg, D. W., Bahcall, N. A., Brinkmann, J., Britton, M., Connolly, A. J., Csabai, I., Fukugita, M., Loveday, J., Meiksin, A., Munn, J. A., Nichol, R. C., Okamura, S., Quinn, T., Schneider, D. P., Shimasaku, K., Strauss, M. A., Tegmark, M., Vogeley, M. S., Weinberg, D. H., Aug. 2003. The Galaxy Luminosity Function and Luminosity Density at Redshift $\mathrm{z}=0.1$. ApJ592, 819-838.

Blanton, M. R., Roweis, S., Feb. 2007. K-Corrections and Filter Transformations in the Ultraviolet, Optical, and Near-Infrared. AJ133, 734-754.

Blanton, M. R., Schlegel, D. J., Strauss, M. A., Brinkmann, J., Finkbeiner, D., Fukugita, M., Gunn, J. E., Hogg, D. W., Ivezić, Ž., Knapp, G. R., Lupton, R. H., Munn, J. A., Schneider, D. P., Tegmark, M., Zehavi, I., Jun. 2005. New York University Value-Added Galaxy Catalog: A Galaxy Catalog Based on New Public Surveys. AJ129, 2562-2578.

Blumenfeld, R., Mandelbrot, B. B., Jul 1997. Lévy dusts, mittag-leffler statistics, mass fractal lacunarity, and perceived dimension. Phys. Rev. E 56, $112-118$.

URL http://link.aps.org/doi/10.1103/PhysRevE.56.112

Bondi, H., 1947. Spherically symmetrical models in general relativity. MNRAS107, 410.

Borgani, S., Feb. 1993. The multifractal behaviour of hierarchical density distributions. MNRAS260, 537-549. 
Capozziello, S., Funkhouser, S., 2009. Fractal Large-Scale Structure from a Stochastic Scaling Law Model. Modern Physics Letters A 24, 1743-1748.

Caruso, F., Oguri, V., Mar. 2009. The Cosmic Microwave Background Spectrum and an Upper Limit for Fractal Space Dimensionality. ApJ694, 151153.

Célérier, M.-N., Jan. 2000. Do we really see a cosmological constant in the supernovae data? A\&A353, 63-71.

Chacón-Cardona, C. A., Casas-Miranda, R. A., Dec. 2012. Millennium simulation dark matter haloes: multifractal and lacunarity analysis and the transition to homogeneity. MNRAS427, 2613-2624.

Coleman, P. H., Pietronero, L., Jun. 1992. The fractal nature of the universe. Physica A Statistical Mechanics and its Applications 185, 45-55.

Durrer, R., Sylos Labini, F., Nov. 1998. A fractal galaxy distribution in a homogeneous universe? A\&A339, L85-L88.

Enqvist, K., Feb. 2008. Lemaitre Tolman Bondi model and accelerating expansion. General Relativity and Gravitation 40, 451-466.

Gabrielli, A., Sylos, Joyce, M., Pietronero, L., 2005. Statistical Physics for Cosmic Structures. Springer Verlag.

Gaite, J., 2005. The fractal distribution of haloes. EPL (Europhysics Letters) $71(2), 332$.

URL http://stacks.iop.org/0295-5075/71/i=2/a=332

Gaite, J., Mar. 2007. Halos and Voids in a Multifractal Model of Cosmic Structure. ApJ658, 11-24.

Grujić, P. V., Oct. 2003. The concept of a hierarchical cosmos. Publications de l'Observatoire Astronomique de Beograd 75, 257-262.

Hamuy, M., Nov. 2013. The acceleration of the Universe in the light of supernovae - The key role of the Cerro Tololo Inter-American Observatory. ArXiv e-prints. 
Hamuy, M., Trager, S. C., Pinto, P. A., Phillips, M. M., Schommer, R. A., Ivanov, V., Suntzeff, N. B., Sep. 2000. A Search for Environmental Effects on Type IA Supernovae. AJ120, 1479-1486.

Hausdorff, F., 1918. Dimension und ueres ma. Mathematische Annalen 79, 157-179, 10.1007/BF01457179.

URL http://dx.doi.org/10.1007/BF01457179

Hogg, D. W., Eisenstein, D. J., Blanton, M. R., Bahcall, N. A., Brinkmann, J., Gunn, J. E., Schneider, D. P., May 2005. Cosmic Homogeneity Demonstrated with Luminous Red Galaxies. ApJ624, 54-58.

Humphreys, N. P., Matravers, D. R., Maartens, R., Oct. 1998. Exact isotropic cosmologies with local fractal number counts. Classical and Quantum Gravity 15, 3041-3049.

Joyce, M., Sylos Labini, F., Gabrielli, A., Montuori, M., Pietronero, L., Nov. 2005. Basic properties of galaxy clustering in the light of recent results from the Sloan Digital Sky Survey. A\&A443, 11-16.

Kobayashi, N., Yamazaki, Y., Kuninaka, H., Katori, M., Matsushita, M., Matsushita, S., Chiang, L.-Y., Jul. 2011. Fractal Structure of Isothermal Lines and Loops on the Cosmic Microwave Background. Journal of the Physical Society of Japan 80 (7), 074003.

Komatsu, E., Dunkley, J., Nolta, M. R., Bennett, C. L., Gold, B., Hinshaw, G., Jarosik, N., Larson, D., Limon, M., Page, L., Spergel, D. N., Halpern, M., Hill, R. S., Kogut, A., Meyer, S. S., Tucker, G. S., Weiland, J. L., Wollack, E., Wright, E. L., Feb. 2009. Five-Year Wilkinson Microwave Anisotropy Probe Observations: Cosmological Interpretation. ApJS180, 330-376.

Longair, M., 2008. Galaxy formation. Astronomy and astrophysics library. Springer.

URL http://books.google.com.co/books?id=e-wJHSBOuZAC

Mandelbrot, B., 1983. The fractal geometry of nature. W.H. Freeman. URL http://books.google.es/books?id=0R2LkE3N7-oC

Mandelbrot, B. B., Dec. 1997. Galaxy distributions and fractals. Astrophysical Letters and Communications 36, 1-5. 
Martínez, V., Saar, E., Dec. 2002. Clustering statistics in cosmology. In: J.-L. Starck \& F. D. Murtagh (Ed.), Society of Photo-Optical Instrumentation Engineers (SPIE) Conference Series. Vol. 4847 of Society of PhotoOptical Instrumentation Engineers (SPIE) Conference Series. pp. 86-100.

Martínez, V., Saar, E., 2002. Statistics of the galaxy distribution. Chapman \& Hall/CRC.

URL http://books.google.com.co/books?id=f-19bB-7AQMC

Mittal, A., Lohiya, D., 2003. Fractal dust model of the universe based on Mandelbrot's conditional cosmological principle and general theory of relativity. Fractals 11, 145-153.

Muñoz-Cuartas, J. C., Mueller, V., May 2012. Galaxy groups and haloes in the SDSS-DR7. MNRAS423, 1583-1595.

Nakamichi, A., Morikawa, M., Oct. 2004. Is galaxy distribution non-extensive and non-Gaussian? Physica A Statistical Mechanics and its Applications $341,215-233$.

Peacock, J., 1999. Cosmological physics. Cambridge University Press.

URL http://books.google.com.co/books?id=t80-yylU0j0C

Perlmutter, S., Aldering, G., Goldhaber, G., Knop, R. A., Nugent, P., Castro, P. G., Deustua, S., Fabbro, S., Goobar, A., Groom, D. E., Hook, I. M., Kim, A. G., Kim, M. Y., Lee, J. C., Nunes, N. J., Pain, R., Pennypacker, C. R., Quimby, R., Lidman, C., Ellis, R. S., Irwin, M., McMahon, R. G., Ruiz-Lapuente, P., Walton, N., Schaefer, B., Boyle, B. J., Filippenko, A. V., Matheson, T., Fruchter, A. S., Panagia, N., Newberg, H. J. M., Couch, W. J., Project, T. S. C., Jun. 1999. Measurements of $\Omega$ and $\Lambda$ from 42 High-Redshift Supernovae. ApJ517, 565-586.

Provenzale, A., Spiegel, E. A., Thieberger, R., Mar. 1997. Cosmic lacunarity. Chaos 7, 82-88.

Ribeiro, M. B., Mar. 1992. On modeling a relativistic hierarchical (fractal) cosmology by Tolman's spacetime. I - Theory. ApJ388, 1-8.

Ribeiro, M. B., Jan. 2005. Cosmological distances and fractal statistics of galaxy distribution. A\&A429, 65-74. 
Riess, A. G., Filippenko, A. V., Challis, P., Clocchiatti, A., Diercks, A., Garnavich, P. M., Gilliland, R. L., Hogan, C. J., Jha, S., Kirshner, R. P., Leibundgut, B., Phillips, M. M., Reiss, D., Schmidt, B. P., Schommer, R. A., Smith, R. C., Spyromilio, J., Stubbs, C., Suntzeff, N. B., Tonry, J., 1998. Observational evidence from supernovae for an accelerating universe and a cosmological constant. The Astronomical Journal 116 (3), 1009.

URL http://stacks . iop.org/1538-3881/116/i=3/a=1009

Sarkar, P., Yadav, J., Pandey, B., Bharadwaj, S., Oct. 2009. The scale of homogeneity of the galaxy distribution in SDSS DR6. MNRAS399, L128L131.

Scrimgeour, M., Davis, T., Blake, C., James, J. B., Poole, G., et al., 2012. The WiggleZ Dark Energy Survey: the transition to large-scale cosmic homogeneity. Mon.Not.Roy.Astron.Soc. 425, 116-134.

Springel, V., White, S. D. M., Jenkins, A., Frenk, C. S., Yoshida, N., Gao, L., Navarro, J., Thacker, R., Croton, D., Helly, J., Peacock, J. A., Cole, S., Thomas, P., Couchman, H., Evrard, A., Colberg, J., Pearce, F., Jun. 2005. Simulations of the formation, evolution and clustering of galaxies and quasars. Nature435, 629-636.

Sylos Labini, F., Vasilyev, N. L., Pietronero, L., Baryshev, Y. V., May 2009. Absence of self-averaging and of homogeneity in the large-scale galaxy distribution. EPL (Europhysics Letters) 86, 49001.

Uchaikin, V. V., Jun. 2004. If the Universe Were a Levy-Mandelbrot Fractal. Gravitation and Cosmology 10, 5-24.

Verevkin, A. O., Bukhmastova, Y. L., Baryshev, Y. V., Apr. 2011. The non-uniform distribution of galaxies from data of the SDSS DR7 survey. Astronomy Reports 55, 324-340.

Wald, R., 2010. General Relativity. University of Chicago Press. URL http://books.google.com.co/books?id=9S-hzg6-moYC

Yadav, J. K., Bagla, J. S., Khandai, N., Jul. 2010. Fractal dimension as a measure of the scale of homogeneity. MNRAS405, 2009-2015. 
Yang, X., Mo, H. J., van den Bosch, F. C., Pasquali, A., Li, C., Barden, M., Dec. 2007. Galaxy Groups in the SDSS DR4. I. The Catalog and Basic Properties. ApJ671, 153-170. 